\title{
An Uncommon Disease Presenting as a Common One Emergency Department Practice Challenges in COVID 19 Era
}

\author{
Abdullah Al-Shamrani, Ahmed A. Alsamman, Mohammed A. Hasabalnabi
}

Prince Sultan Military Medical City (PSMMC)

Corresponding author: Ahmed A. Alsamman, email: aasamman@ hotmail.com, Mobile: 0966565575297

\begin{abstract} another point of consideration that consume time and effort. malignancy.

\section{CASE REPORT}

History: a 12 years old girl, previously healthy, was referred from primary care to ED at Prince Sultan Military Medical City complaining of fever and tachypnea. Nine days earlier, the girl sought advice to ED complaining of chest pain and headache. Her symptoms were attributed to myositis because pain was localized and chest wall was tender on palpation. She was discharged on analgesics that relieved her symptoms. There was no weight loss or night sweats. She didn't have any COVID 19 epidemiological risk factors. However, she was put in isolation ward.
\end{abstract}

Background: doctors working in Emergency Department (ED) face the challenge of persistently applying practice guidelines and evidence-based medicine. In addition, physicians consider other factors such as bed vacancies, administrative rules and above all, medicolegal problems. In COVID 19 era, strict infection control precautions rose as

Objective: ED physicians must show flexibility when they follow guidelines, especially during the current pandemic. Results: the patient presenting with clinical picture suggestive of pneumonia was finally diagnosed with serious

Keywords: Chest mass, Community acquired pneumonia (CAP), COVID 19, Emergency Department (ED).

Examination: the child was fully conscious and cooperative but she was obviously tachypneic. Weight and height were at $50^{\text {th }}$ percentile. Vital signs showed a respiratory rate $(\mathrm{RR})$ of $43 /$ minute, heart rate was 140 , temperature of $39.4^{\circ} \mathrm{C}$, normal blood pressure of $111 / 65$ , and her pulse oximeter read 97\%. On auscultation, she had normal vesicular air entry with no bronchial breathing or friction rub. Right side showed decreased air entry. There was no working accessory muscles of respiration. Later, after fever had subsided, (RR) was still 43/minute while heart rate decreased to 120 . Cardiovascular exam was normal except for tachycardia. There was no associated lymphadenopathy or organomegaly. Examination of other systems was unremarkable. Throat exam was not performed for fear of aerosol generation abiding with infection control precautions.

The provisional diagnosis was community-acquired pneumonia.

Question 1: the ED doctor faced this decision making challenge. Whether to follow national ${ }^{(1)}$ and western ${ }^{(2-4)}$ guidelines as regard community acquired pneumonia, and treat the girl with oral antibiotics and send her home without any laboratory or radiological investigations except PCR for COVID19 or to admit in ED for further investigation, initial intravenous antibiotics and possible admission to the ward?

The ED doctor took the second choice and investigations were done.

\section{Investigation}

- Laboratory result showed: WBC 8,900 10^9/1, HB $9.5 \mathrm{~g} / \mathrm{dl}$ and PLT. 848,000 10^9/1

- $\quad$ PT $19.1 \mathrm{sec}$, PTT $44 \mathrm{sec}$, INR 1.4,

- Fibrinogen $7.8 \mathrm{~g} / \mathrm{L}$

- Ferritin 94; ESR $102 \mathrm{~mm} / \mathrm{hr} \mathrm{C}$ - reactive protein 486 $\mathrm{mg} / \mathrm{L}$

- Procalcitonin $0.76 \mathrm{ng} / \mathrm{ml}$. D-Dimer 9.440 .000 $.500 \mathrm{ug} / \mathrm{ml}$

- COVID PCR came negative later.

- X-rays (Fig $1 \mathrm{a}$ and b) showed right side opacity with no rising fluid level to the axilla. 


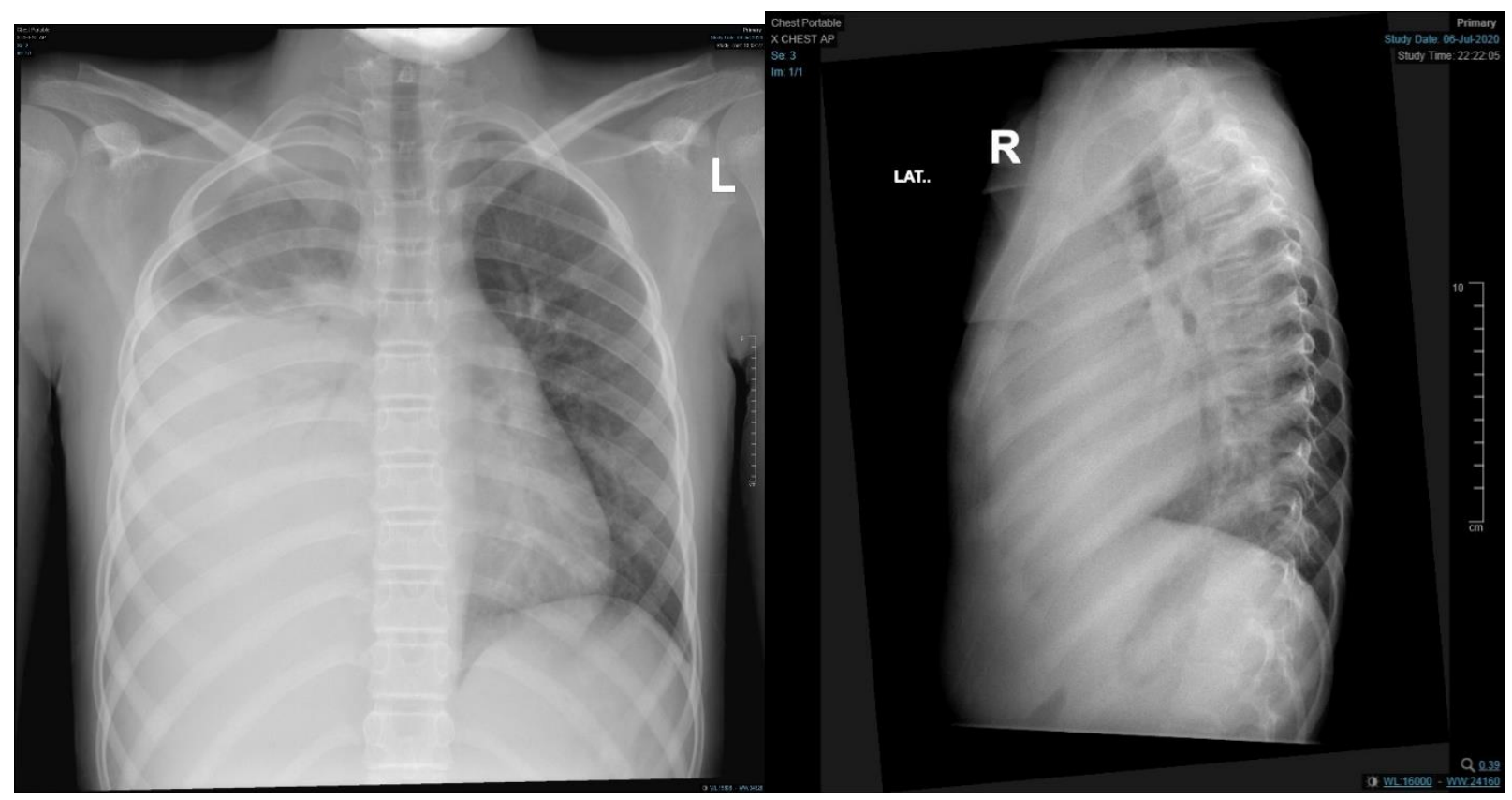

(a)

(b)

Fig 1a: AP film showing homogenous opacity involving more than two third of right middle and lower zone obscuring cardiac border, cardiophrenic border and costophrenic angle. There was no significant tracheal deviation to any side.

Fig 1b: lateral film showing opacity involving anterior chest.

Question 2: the working diagnosis was still CAP. Based on the investigations done, should doctors had focused on COVID 19? If so, what to do?

The question has no clinical relevance because management of COVID pneumonia in pediatric age group was not well established yet. Nevertheless, the ED doctor decided to admit under general pediatric for treatment with double antibiotics (ceftriaxone and vancomycin), fluids, analgesics with close observation and potential further evaluation of the lung opacity.

Further evaluation: The girl was admitted as a case of community-acquired pneumonia with a possibility of COVID 19 infection. Detailed history taking unveiled some useful information. The girl has been complaining of chest pain for a longer time than what was previously mentioned, nearly one month. In addition, there was subjective weight loss over the same period.

Further investigation: During admission, chest ultrasonography (Fig. 2) requested showing surprisingly minimal effusion with no septation, not matching the opacity. Therefore, pulmonologists were consulted and computed tomography (Fig. 3) was requested for detailed description of the right lung field. The result showed an extrapulmonary right pleural complex mass measuring approximately $9 \times 10 \times 11 \mathrm{~cm}$ in dimension seen in the lower aspect of the right hemithorax. Finally, oncology team was involved and with the help of intervention radiology, a biopsy was performed confirming a diagnosis of Ewing sarcoma.

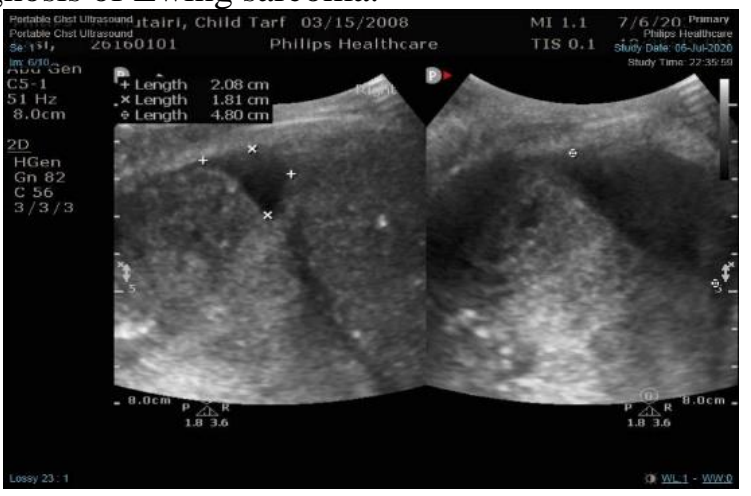

Fig. 2: large non homogenous lobulated soft tissue echogenicity in right lower chest.

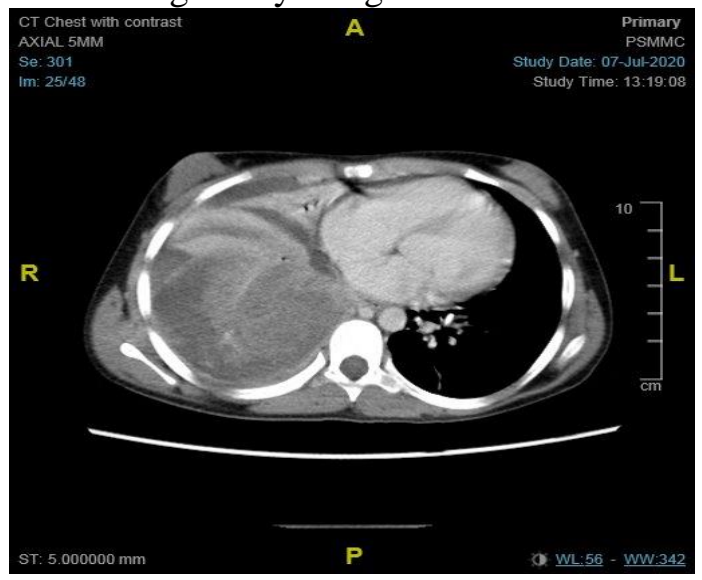

Fig. 3: Extrapulmonary right complex mass. 


\section{DISCUSSION}

On the first hand, primary pleural tumors are benign or malignant lesions arising from either the parietal or visceral pleura. They are very rare ${ }^{(5)}$. Therefore, diagnosis of such tumors needs high index of suspicion. Furthermore, extensive radiological and histopathological work up is needed ${ }^{(6)}$.

On the other hand, CAP is a quite common disease in children that is caused by different bacteria and viruses (7). CAP frequently jumps to the minds of ED physicians. Diagnosis of CAP in school aged children is mainly a clinical one and ordering chest radiographs is not a must (3). Treatment of CAP in the same age group is by oral antibiotics although admission is needed in certain situations ${ }^{(1,2)}$.

\section{RECOMMENDATIONS}

Although evidence-based guidelines have become a corner stone in current medical practice, physicians, especially ED doctors should tailor plans for patients under certain circumstances.

- Although COVID19 pandemic had its effect on the entire globe, physicians should anticipate other serious medical condition.

- Children reporting chest pain during multiple medical visits should be cautiously investigated even if the pain is mild and self-limited .

- ED physicians may miss some details during history taking, which is a pitfall that needs to be treated.

\section{ETHICAL APPROVAL}

Signed informed consent for participation and publication of medical details was obtained from the child's parents. Confidentiality of patients data were ensured in all stages.

The authors declare that ethics approval was taken for this case report.

\section{CONFLICT OF INTEREST}

The authors declare that there is no conflict of interest regarding the publication of this article.

\section{FUNDING}

None.

\section{CONSENT FOR PUBLICATION}

Written informed consent was obtained from the participants.

\section{REFERENCES}

1. Alzomor O, Alhaggar S, ALjubair F et al. (2017) : Management of community-acquired pneumonia in infants and children: Clinical practice guidelines endorsed by the Saudi Pediatric Infectious Diseases Society. International Journal of Pediatrics and Adolescent Medicine, $4: 153-158$.

2. Hayes B and George C (2012): Community-Acquired Pneumonia in Children.Am Fam Physician, 86 (7):661667.

3. National Institute for Health and Care Excellence (2014): Pneumonia (including community acquired pneumonia). http://www.nice. org.uk/guidance/cg1913.

4. Harris M, Clark J, Coote N et al. (2011): British Thoracic Society Guidelines for Management of Community Acquired Pneumonia in Children. Thorax, 6:1-23.

5. Dela Cruz C (2011): Lung cancer: epidemiology, etiology, and prevention. Clin Chest Med., 32: 605-44.

6. 6 Christopher T, Kelsey M, Anthony W (2013): Rare Pleural Tumors. Clin Chest Med., 34 113-136

7. Michelow I, Olsen K, Lozano J et al. (2004): Epidemiology and clinical characteristics of communityacquired pneumonia in hospitalized children. Pediatrics, 113(4):701-7. 